\title{
Honey Bees Repellent Device: Preliminary Experimental Research with the Bees Hearing Sensitivity
}

\author{
${\text { David } \operatorname{Costa}^{1(\bowtie)} \text {, João Paulo Teixeira }}^{1,2}$, and Vasco Cadavez ${ }^{3}$ \\ ${ }^{1}$ CEDRI - Research Centre in Digitalization and Intelligent Robotics, \\ Instituto Politécnico de Bragança (IPB), Bragança, Portugal \\ \{dcosta, joaopt\}@ipb.pt \\ ${ }^{2}$ UNIAG - Applied Management Research Unit, \\ Instituto Politécnico de Bragança (IPB), Bragança, Portugal \\ ${ }^{3}$ CIMO Mountain Research Centre, School of Agriculture, \\ Instituto Politécnico de Bragança (IPB), Bragança, Portugal \\ vcadavez@ipb.pt
}

\begin{abstract}
Bees are insects that attack, to protect the hive, when they feel threatened. The main objective in this paper was to build an electronic device capable of repelling bees. Thus, a study of the hearing thresholds, of honey bees, has been developed to find out the frequencies range are most sensitive. This knowledge can be important to identify a frequency or a sound capable of repealing them. We also present an electronic circuit developed to build a repelling device able to reproduce a recorded sound or periodic sound. We report also a series of laboratory behaviour experiments, where honey bees (Apis mellifera spp.) had to make the choice between a box where a sound was being played or another box without sound. The experiments were conducted using the following sound frequencies: 100, 150, 200, 300, 400, 500 and $550 \mathrm{~Hz}$; and also, with the sound of three natural predators: the drone, the swallow and the Asian wasp. The honey bees used in the experiments were previously conditioned to go to the box with sound that contained food in order to associate the sound to the presence of food.
\end{abstract}

Keywords: Repellent device $\cdot$ Pure tones $\cdot$ Bees hearing $\cdot$ Bees predators

\section{Introduction}

Honey bees are social insects living in a hive with thousands of individuals, mainly female workers. Each one of the individuals has its specific role to play. However, the queen has key role in the hive, since she is responsible for the harmony of the colony, and is also responsible for the reproduction, living up to six years. The drones have as unique purpose the fertilization of the queen, and they live to a maximum of three months, and those who succeed to copulate with the queen die. The working bees are responsible for the maintenance and hygiene of the hive, and for the food and water supply for all who live inside the hive by collecting pollen and nectar. They also 
produce wax for the combs and defend the hive from any predators. They can live up to six months.

For a long time, it was believed that honey bees were deaf; but this theory was refuted by the experiments from Towne and Kirchner [1] who discovered that the dancing bees emit sound signals. These authors succeed that honey bees to associate a sound with a weak electric shock; and they learned to avoid that shock by leaving the feeder when a sound was given. Thus, they concluded that honey bees can hear airborne sound. Another study was conducted, under a different paradigm, where individual honey bees were trained to make a choice to turn right or left in a Y-shaped labyrinth, when entering a feeder, the correct way being toward the sound source. In this case it was concluded that honey bees can learn that food is always presented associated to a sound [2].

Honey bees pick up the vibrations of a sound by converting it from mechanical vibrations to nerve impulses that are transmitted to the brain [3]. The antennae (Fig. 1) is one of the most important organ in bees hearing because it is there that Johnston's organ is situated and it has also mechanosensory hairs which helps in hearing [4].

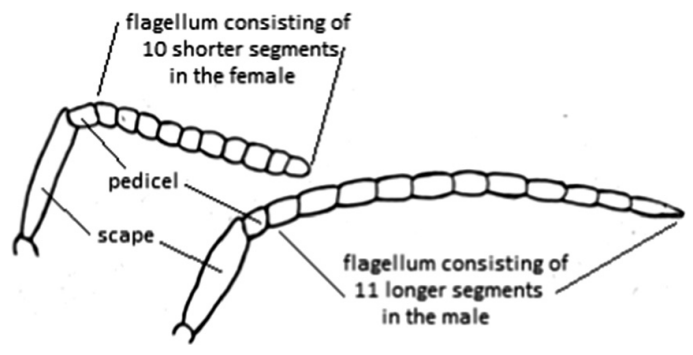

Fig. 1. Antennae [6].

The Johnston's organ, situated at the base of the antennae as shown in Fig. 2 receives the mechanical vibrations of a sound and converts it into nervous impulses which are transmitted to the brain [4]. According to Dreller and Kirchner [5], to test if the existent mechanosensory hairs in the antennae help in hearing they put a small silicon tube around the pedicel of one of the antennas and then trained to react to a sound with a frequency of $256 \mathrm{~Hz}$.

Honey bees are known by its capacity of associative learning both in the field and in laboratory-controlled conditions. They can learn to remember odours, shapes and sounds; they can also be trained in classic and conditioned paradigms [8]. In classic conditioning, honey bees learn to associate a stimulus to biological relevant stimulus, while in operant conditioning, they evaluate its own behavior and consequences [9].

The main purpose of this work was to understand if there is a frequency band or a sound that can repel bees in order to build an electronic device capable of keeping them away. The potential of this device would be high and of interest to the general population as it would serve as a sustainable alternative to fumigators and could be used by all who are allergic to bee sting. 


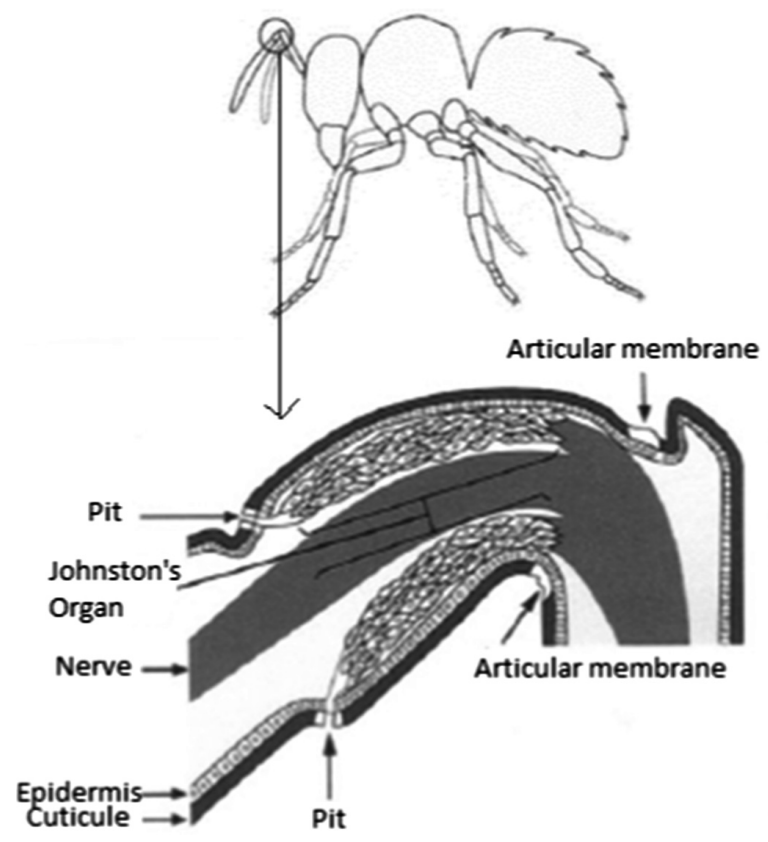

Fig. 2. Johnston's Organ [7].

In this paper, we present a series of experiments using several sound frequencies and sound of honey bee's predators to condition the honey bees to a sound in the presence of food in a Y-shaped labyrinth. The main goals were to determine the upper and lower thresholds of a honey bees hearing, and their reaction facing the frightening sound of a predator.

\section{Materials and Methods}

Some boxes were built to keep, transport and conduct the experiments; and electronic counters were developed to count the number of honey bees who went to each box. Three preliminary experiments were performed to observe honey bees' behaviour and find the most correct method to keep them so they would have a life period more extended in a laboratory environment.

In order to simplify the process of counting honey bees entering each box, a counter was constructed using an Arduino UNO and an LDR. The counter was placed in the inlet of boxes 1 and 2 in a plastic tube. Figure 3 shows the connections made to Arduino and to the LCD. This system works by sensing the intensity of light in its environment.

The LDR gives out an analog voltage when connected to Vcc $(5 \mathrm{~V})$, which varies in magnitude in direct proportion to the input light intensity on it. The LDR is connected to the analog input pin on the Arduino. The Arduino, with its built-in analog to 
digital converter (ADC), converts it (from $0-5 \mathrm{~V}$ ) into a digital value in the range of 0 1023 (10 bits resolution). When there is sufficient light in its environment or on its surface, the converted digital values read from the LDR through the Arduino will be in the range of $800-1023$.

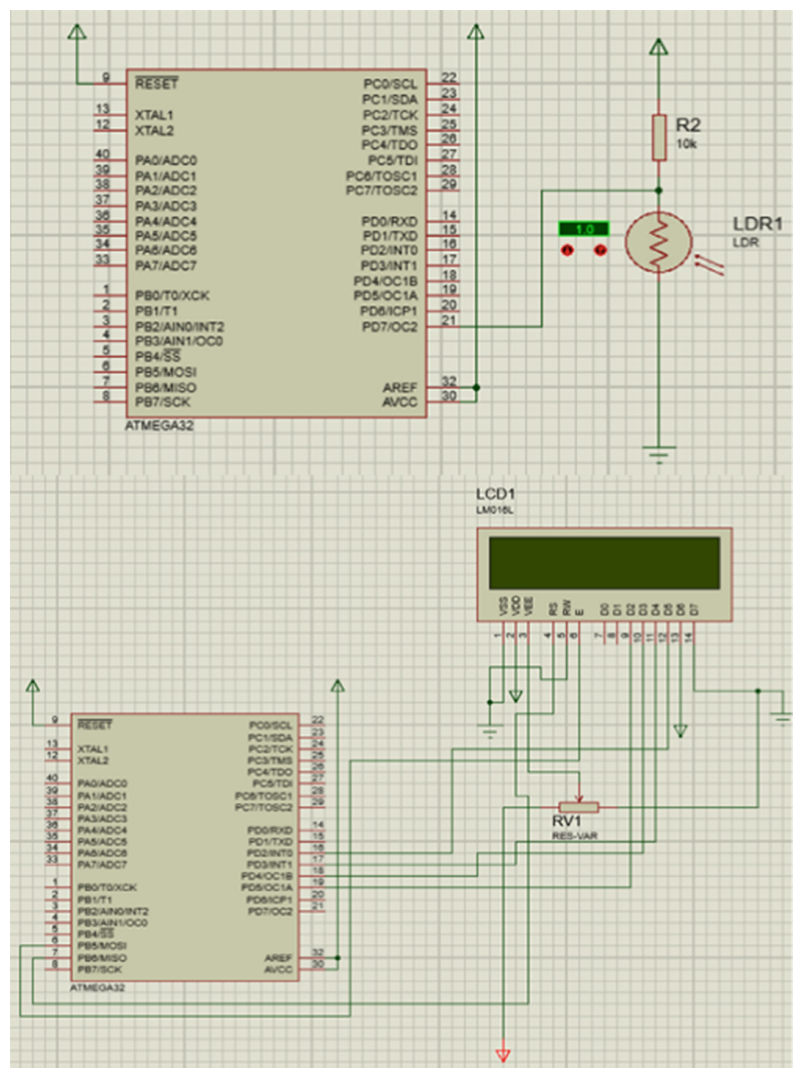

Fig. 3. Arduino and LCD connections.

\section{System of Boxes to Transport and Maintain Honey Bees}

To keep the honey bees in the laboratory, three boxes were built and another one to transport them from the apiary to the laboratory. The main box, together with the two small boxes, constituted a Y-shaped system where experiments were performed (Fig. 4). At the beginning of each experiment, the honey bees were all placed in the main box, and then the access to the small boxes was simultaneously opened so that the honey bees would have a choice to enter to search for food. In one of the small boxes a pure tone was placed, after they had been conditioned with the same sound and with food. 


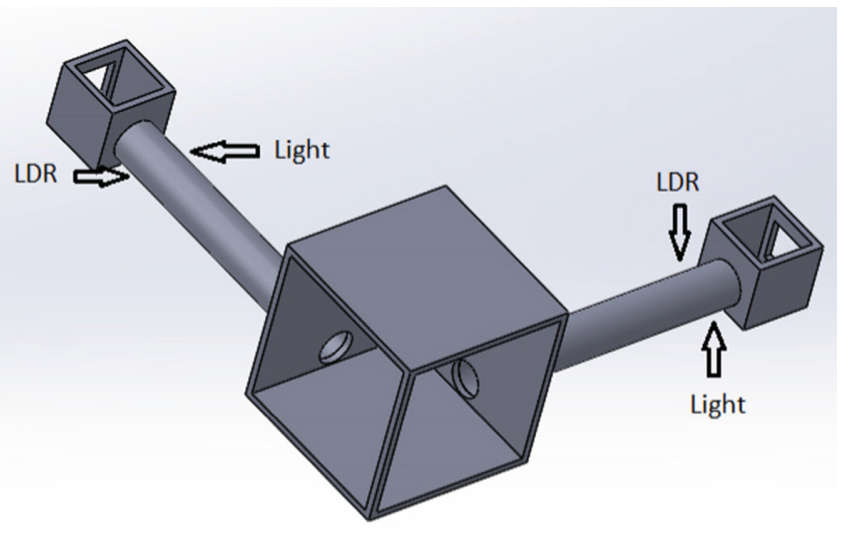

Fig. 4. System of boxes.

\subsection{Main Box}

The main box with $50 \times 50 \times 50 \mathrm{~cm}$ was built with PVC plates and two tubes with $1 \mathrm{~m}$ to make the connection to the small boxes. The tubes are removable and in the holes of the tube connections there are "doors" that slide up and down when it is necessary to remove the tubes.

\subsection{Conveyor Box}

The conveyor box was used to transport the honey bees from the apiary to the laboratory. The box with $20 \times 20 \times 20 \mathrm{~cm}$ was built with PVC plates and a door was made that moves up and down with a five-centimeters fitting the entrance of the hive.

\subsection{Small Boxes}

The two small boxes with $15 \times 15 \times 15 \mathrm{~cm}$ were made with four PVC plates each, one door at the rear and the top part was coated with acetate sheet. The tube connects the main box to the small ones with the entry in these boxes being flattened (for the purpose of introducing the counter device). In the back plate, a square was cut and it was covered with net to place the loud speaker. This boxes system gives the honey bees two options, one exit with sound and the other without sound.

\section{Repellent Device}

For the construction of the device to ward off honey bees, it would be necessary to find a sound or a tone that would be threatening to bees and that when they heard that sound, it caused them to turn away.

This device could be done based on the NE555 integrated circuit (for threatening periodic sound) that is a precision timing circuit capable of producing accurate time 
delays or oscillation. The NE555 can be used to provide time delays, as an oscillator and as a flip-flop element. The standard NE555 package includes 25 transistors, 2 diodes and 15 resistors. This IC can work in three operating modes:

- Astable (free-running) mode - it operates as an electronic oscillator;

- Monostable mode - in this mode, the NE555 functions as a "one-shot" pulse generator;

- Bistable (schmitt trigger) mode - the NE555 can operate as a flip-flop.

The NE555 produces square waves instead of sinusoids, meaning that it has harmonics which do not matter much because they have one main component that is higher than its harmonics. The harmonics are shown by the following equation for the time-series Fourier transform coefficient, $\mathrm{C}_{\mathrm{k}}$ :

$$
C_{k}=\frac{1}{k \pi}
$$

when $\mathrm{k}$ is even, then $\cos \mathrm{k} \pi=1$ so $\mathrm{C}_{\mathrm{k}}=0$;

when $\mathrm{k}$ is odd, then $\cos \mathrm{k} \pi=-1$, so $C_{k}=\frac{2}{k \pi}$, therefore there is only odd harmonic components, as described by Fig. 5 .

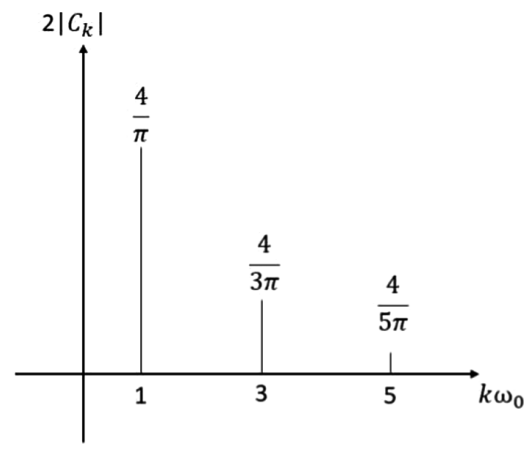

Fig. 5. Magnitude Spectrogram of the squared wave.

The frequency can be controlled using three resistors and a capacitor (R1, R2, R3) with the following equation:

$$
f=\frac{1}{\ln (2) \cdot C \cdot(R 1+2 R 2)}
$$

In case the sound that causes the bee's withdrawal is a frequency, Fig. 6 shows an example of a circuit that can produce it.

In case the sound is not a frequency, it can be recorder and reproduced by the integrated circuit ISD1700. This circuit is a high-quality chip that allows recording. 


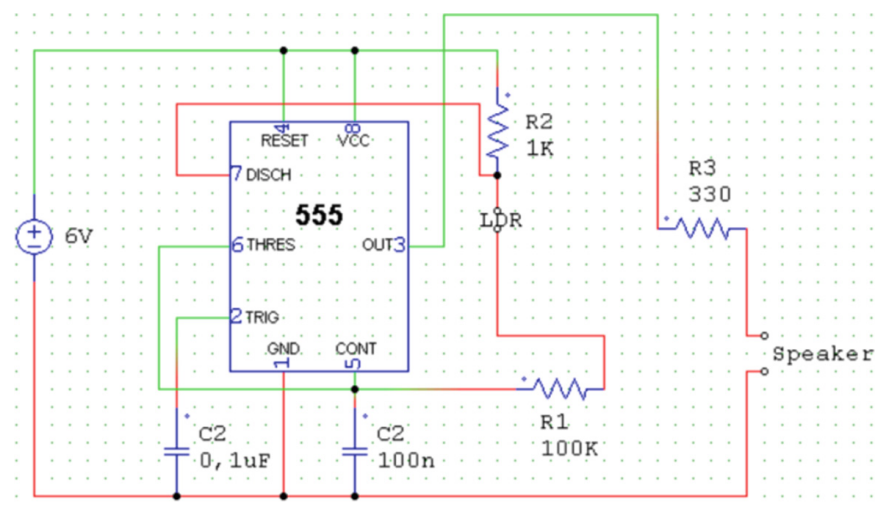

Fig. 6. Circuit to produce symmetric periodic squared waves.

The duration of recording can be as much as $120 \mathrm{~s}$ by altering the sampling rate between $4 \mathrm{kHz}$ to $11 \mathrm{kHz}$ and that frequency is altered by an external resistor.

Recordings are stored into on-chip Flash memory, providing zero-power message storage. This unique single-chip solution is made possible through Winbond's patented Multi-Level Storage (MLS) technology. Audio data are stored directly in solid-state memory without digital compression, providing superior quality voice and music reproduction.

The circuit in Fig. 7 shows a possible way to create the proposed device.

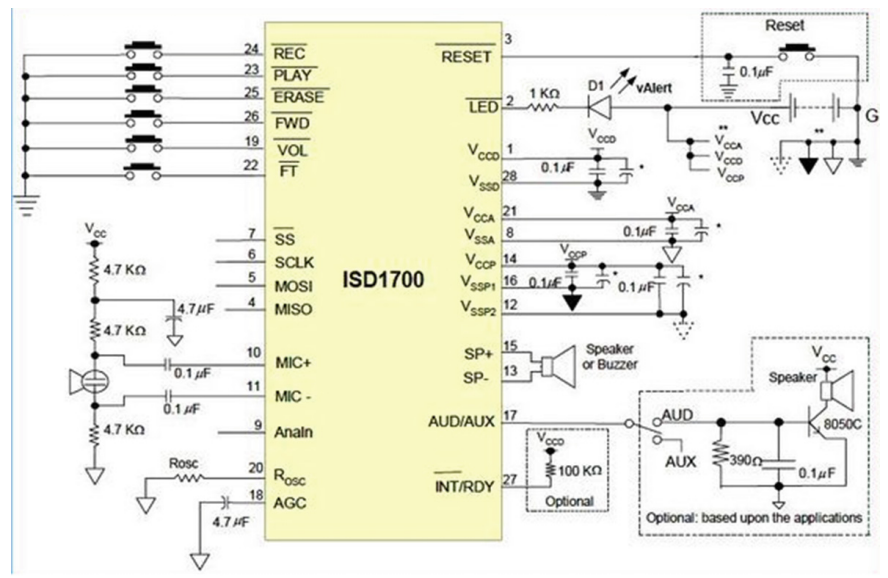

Fig. 7. Circuit of the proposed device [10]. 


\section{Experiments and Results}

All experiments were done after the honey bees were between 50 to $60 \mathrm{~min}$ without food, as shown in Fig. 8. So, they would search for the feeder when the accesses to the tubes/boxes were simultaneously opened. One of the box will have the sound but no food. Thus, several experiments with different pure tone frequencies could allow to determining the upper and lower frequency band limits of hearing. Three different types of experiments were implemented, as described below. The first experiments had the purpose to define the setup of next experiments. Second set of experiments was intended to determine the frequency bands of bees hearing and/or some infra-sound or ultra sound that can repel them. The third set of experiments aims to comprehend the reaction of honey bees to the predator sounds.

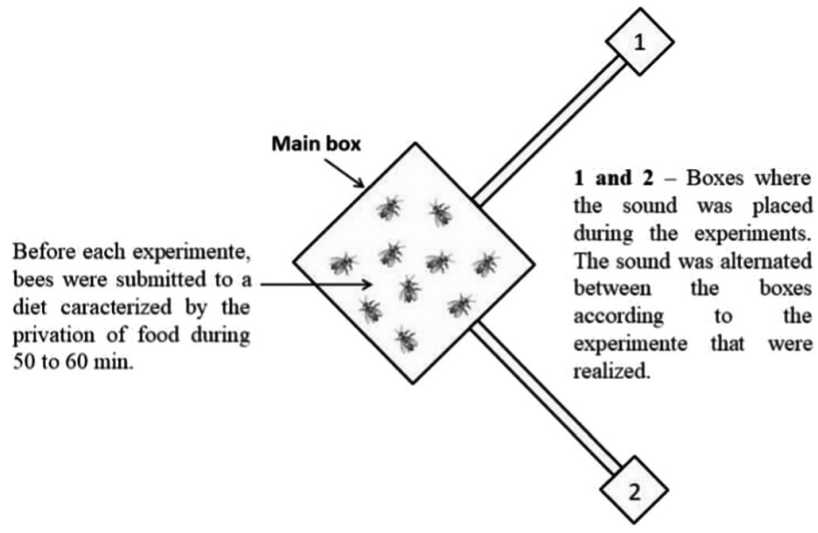

Fig. 8. Experiments scheme.

All experiments were made under temperature and humidity conditions considered adequate $[11,12]$, namely, the temperature was around $30^{\circ} \mathrm{C}$ and $70 \%$ of humidity.

\subsection{Initial Experiments}

The first three experiments had the goal to understand some of the behaviors that honey bees present when subjected to different conditions. The first one, aimed to understand how the honey bees were distributed through the boxes 1 and 2, having only a portion of sucrose in the main box, same temperature and lighting conditions and it was concluded that they distributed similarly by the two boxes. The second one, was carried out with the sucrose only in one of the small boxes to see if the honey bees communicate with each other about the location of the feeder as they do if they were in open field conditions. Finally, the third one was performed to understand the sensitivity of the honey bee to light, the food was placed in boxes 1 and 2 although one of them was covered to be completely dark and the other uncovered. By the results obtained it 
is perceivable that they are strongly sensitive to the light preferring a place with illumination. Therefore, the illuminations conditions should be well controlled in both boxes and all experiments.

In all experiments, the honey bees were previously conditioned to the presence of a sound (pure tones in the various frequencies used).

\section{$1^{\text {st }}$ Experiment}

The first experiment consisted in placing food only in the main box, but with the tube entrances open, and aimed to see how honey bees spread through the two small boxes. From the results obtained it was verified that honey bees distributed equally by the two boxes when they have similar environmental conditions, as shown in Fig. 9.

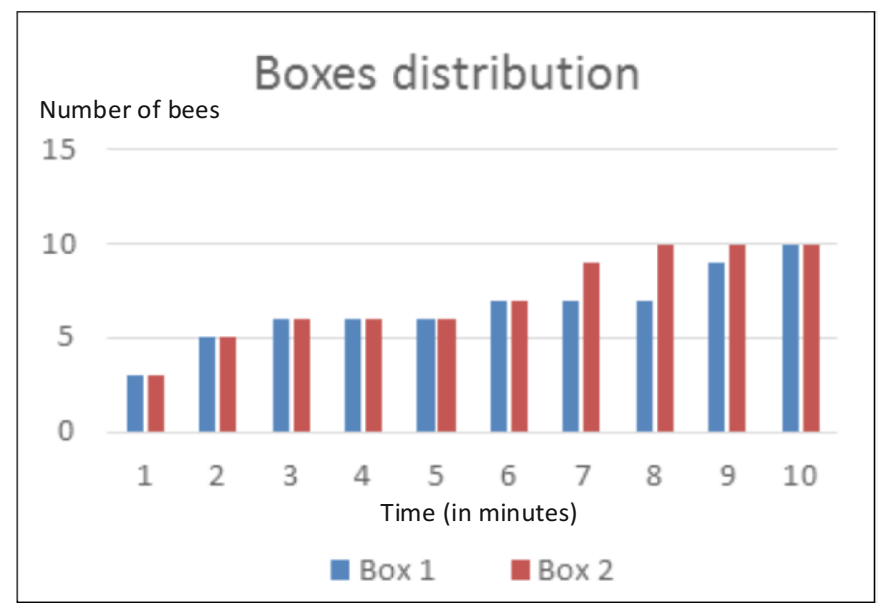

Fig. 9. Boxes distribution experiment.

\section{$2^{\text {nd }}$ Experiment}

In the second experiment, food was placed in one of the small boxes. This experiment had the purpose to verify if the honey bees communicate the food place to others, as they do when they are in a beehive. Figure 10 shows that the honey bees were entering box 1 (containing sucrose) gradually; while in box 2 did not enter none until minute 10 . Therefore, it was assumed honey bees applies some type of communication to point the food location.

\section{$3^{\text {rd }}$ Experiment}

In the third experiment, the sensitivity of the honey bees to the light was verified. The experiment consisted in having one small box in the dark and the other with natural light, both without food. As it can be verified in Fig. 11, honey bees only entered the box with light. Therefore, they are very sensitive to the light. 


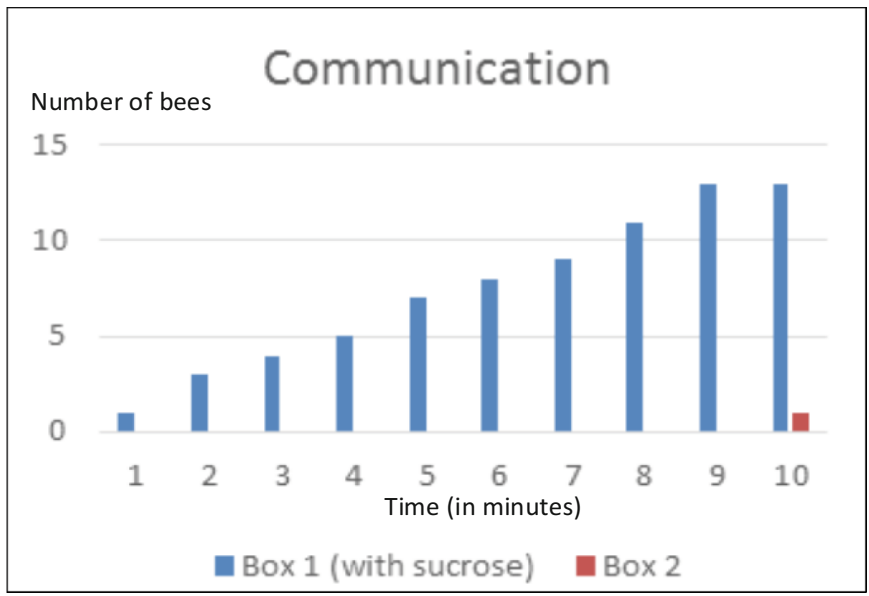

Fig. 10. Communication experiment.

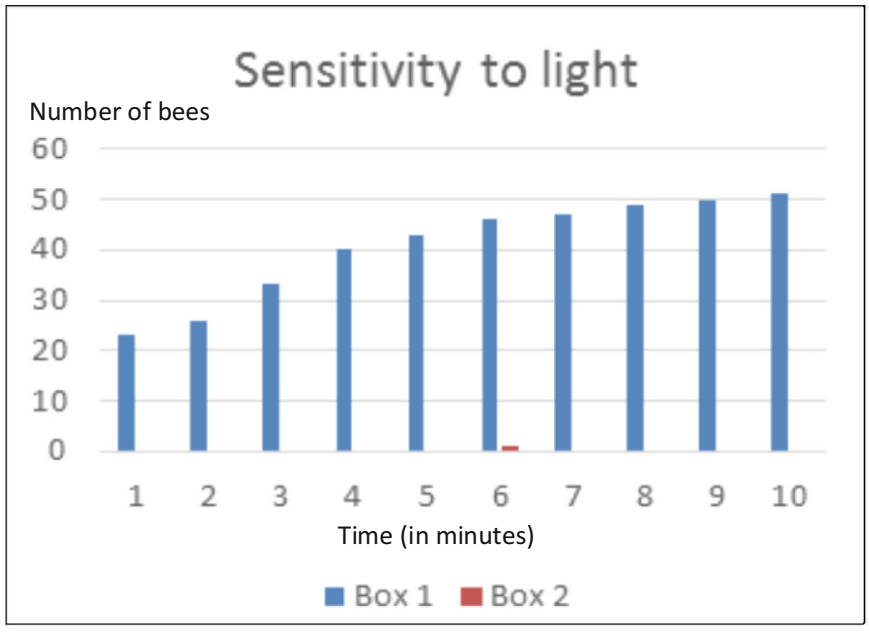

Fig. 11. Light sensitivity experiment.

\subsection{Frequencies Band Hearing Sensitivity}

This section presents a set of experiments with frequencies from $100 \mathrm{~Hz}$ up to $550 \mathrm{~Hz}$ that was realized to find the limits of the audible frequency band by honey bees. Each experiment was done with repetitions, sometimes on different dates and with different groups of honey bees.

In all these experiments the honey bees were previously conditioned with a pure tone with the frequency of the further experiments in the box containing food. 
However, during the experiments no sucrose was placed in any of the boxes to avoid any interference of the smell of the sucrose.

The pure tones were produced with a signal generator with sinusoidal waves with the frequency of the tones connected to a loudspeaker. The volume was controlled in order to be propagated by the small box to the main box by the connecting tube and do not be hearing outside this sound circuit.

Each experience had a duration of 10 min. During this period, the number of bees that entered in each box was registered. The total number of bees inside the main box were not controlled, although there were always between one and two hundred bees. For statistical analysis, the percentage of bees that entered in each box at each repetition was used. The mean value in percentage and the maximum number of bees at each repetition was used for analysis. There were one pair of values for each experiment repetition.

After the experiments were carried out, all the results were submitted to statistical analysis using the Wilcoxon's test. This analysis was performed using the two variants, by the mean and the maximum (in percentage) of honey bees that entered in each box during the $10 \mathrm{~min}$. In Tables 1 and 2, M stands for the weighted mean of the mean/maximum value for all the results of every repetition in every experiment.

For $100 \mathrm{~Hz}$, it was noted that regardless of where the sound was being played, honey bees entered both in box 1 as well as box 2 and the result of the statistical

Table 1. Statistical analysis of experiments with pure tones

\begin{tabular}{|c|c|c|c|c|c|c|}
\hline \multirow{3}{*}{$\begin{array}{l}\text { Frequency } \\
(\mathrm{Hz})\end{array}$} & \multicolumn{3}{|l|}{ Mean } & \multicolumn{3}{|l|}{ Maximum } \\
\hline & \multirow{2}{*}{$\begin{array}{l}\text { Sound/No } \\
\text { sound } \\
\text { P-value }\end{array}$} & \multicolumn{2}{|c|}{ Weighted mean } & \multirow{2}{*}{ 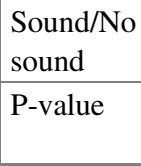 } & \multicolumn{2}{|c|}{ Weighted mean } \\
\hline & & $\begin{array}{l}\text { Sound } \\
\text { (M) }\end{array}$ & $\begin{array}{l}\text { No sound } \\
\text { (M) }\end{array}$ & & $\begin{array}{l}\text { Sound } \\
\text { (M) }\end{array}$ & $\begin{array}{l}\text { No sound } \\
\text { (M) }\end{array}$ \\
\hline 100 & 0,584 & 32,2 & 35,4 & 0,100 & 46,1 & 48,0 \\
\hline 150 & 0,584 & 30,7 & 37,2 & 0,269 & 34,4 & 40,5 \\
\hline 200 & 0,036 & 48,9 & 28,2 & 0,036 & 65,6 & 73,1 \\
\hline 300 & 1,000 & 15,5 & 14,8 & 1,000 & 20,6 & 19,4 \\
\hline 400 & 0,500 & 26,1 & 42,5 & 0,371 & 36,5 & 51,0 \\
\hline 500 & 0,371 & 18,2 & 22,6 & 0,371 & 29,3 & 37,0 \\
\hline 550 & 1,000 & 49,9 & 54,6 & 1,000 & 71,3 & 84,3 \\
\hline
\end{tabular}

Table 2. Statistical analysis of experiments with predator sounds.

\begin{tabular}{l|l|l|l|l|l|l}
\hline \multirow{2}{*}{} & \multicolumn{4}{|l|}{ Mean } & \multicolumn{2}{l}{ Maximum } \\
\cline { 2 - 7 } & \multicolumn{2}{|l|}{ Weighted mean } & \multicolumn{2}{l}{ Weighted mean } \\
\cline { 2 - 7 } & P-value & Sound (M) & No sound (M) & P-value & Sound (M) & No sound (M) \\
\hline Drone & 0,422 & 31,3 & 85,4 & 0,422 & 39,0 & 102,4 \\
\hline Swallow & $\mathbf{0 , 0 3 6}$ & 44,6 & 36,8 & $\mathbf{0 , 0 3 6}$ & 73,0 & 64,4 \\
\hline Asian wasp & 0,059 & 25,1 & 24,6 & 0,059 & 29,8 & 29,3 \\
\hline
\end{tabular}


analysis shows no statistical significance. By the analysis of the results of the experiment and the statistical analysis we can conclude that bees cannot hear a sound with this frequency.

With the frequency of 150, 400 and $500 \mathrm{~Hz}$, there was a clear tendency for more bees to enter the box with sound, like honey bee could hear a sound at this frequency but the statistical analysis shown no statistical significance. It is believed that the reason is the low number of repetitions of each experiment, however these results may serve as an indicator that honey bees can feel these frequencies. It should be mentioned that no more experiments could be made because meanwhile the winter season arrived and only next spring will be possible to repeat the experiments.

For $200 \mathrm{~Hz}$, it was concluded, after the statistical treatment, that the honey bees can hear this frequency $(\mathrm{p}<0.05)$, as can be seen in Table 1. Also, in the analysis of the results of the experiments it was possible to perceive that the honey bees could hear because the number of honey bees counted in the box with sound was much superior in comparison to the box without sound.

For $300 \mathrm{~Hz}$, there is no conclusion that can be drawn since the p-value is equal to 1 . We obtained this value because there were not enough data repetitions.

For $550 \mathrm{~Hz}$, the results are uncertain because more repetitions of the experiments would be necessary, but by the results, before the statistical analysis was applied, there was a tendency for honey bees went in greater number to box 1 , independent were the sound was being played, suggesting that this frequency could not be detected by the honey bees.

\subsection{Predators}

All the following experiments were performed with the sound of three honey bees' predators in order to see if they are repelled in the presence of any of these sounds. The sound produced by the following predators were experimented:

- Drone;

- Swallow;

- Asian wasp.

The sound was reproduced through a loudspeaker in box 1 in two repetitions and in box 2 in another 2 repetitions and the number of honey bees that went to each of the boxes was registered. As in previous experiments, tube accesses were opened simultaneously, in trials 1 and 2 no food was placed inside the boxes and trials 3 and 4 had food in the two boxes so it would be possible to see if there is a difference in the number of honey bees going to each of the boxes when there is food or not.

For the sound of the drone and the Asian wasp, it was possible to conclude that the honey bees do not flee in the presence of this sound. By the statistical treatment applied (resumed in Table 2), none of the cases obtained a statistically significant result $(p>0.05)$. For the sound of the swallow, both for the case of the mean and the maximum, the result was statistically significant. Therefore, the honey bees follow the sound of the swallow, maybe in order to attack the predator to defend the hive.

The lack of statistical significance demands more repetitions of the experiments. 


\section{Conclusions}

This study presents a preliminary result of the behavior of the honey bees concerning its hearing sensitivity. The development of a technologic device to repel the bees could be useful to be used by allergic persons to the bee's sting when been in the country side near to the presence of this insect. This device should produce a sound that could repel the bees and preferable not eared by persons, like infra or ultra sounds.

The search for a sound that could repel the bees was started by some preliminary experiments to establish the setup of the following experiments. This phase allowed also to understand several procedures to improve the life time of the bees under the laboratory conditions, like lightening, heating system, humidity control, the way to supply the sucrose and water. Also, the procedure to condition the bees in order to follow the sound origin to find food was improved along the experiments.

Concerning the sensitivity of the honey bees with the pure tone sounds, it was possible to conclude that at the frequency of $200 \mathrm{~Hz}$, honey bees can hear, but with the other frequencies, there were no confirmation of their sensitivity. In fact, after adequate statistical treatment, it was shown that a greater number of repetitions of the experiments would have been required for each frequency.

Concerning the predator sounds we concluded that in the presence of the sound of the drone and the Asian wasp, bees do not flee whereas for the sound of swallow, the results shows that bees follow its sound.

Although some of the results are inconclusive, there are several indications to proceed with the experiments with more clear directions to have statistical significance on the analysis. The registration of bees entering to the boxes along time were not so useful for the statistical analysis, therefore in next experiments only the total number of bees entering the boxes will be of interest, and many more repetitions should be realized. The experiments would be extended to several infra sound frequencies and ultra-sound frequencies, because non-audible sounds are more interesting for the purpose of the device.

Acknowledgment. The authors would like to thank Prof. Miguel Vilas Boas, for his support and understanding of the honey bees' comportment and behaviour in a natural environment, and the Escola Superior Agrária of the Instituto Politécnico de Bragança, for the support with the honey bees used in the experiments.

This work is supported by the Fundação para a Ciência e Tecnologia (FCT) under the project number UID/GES/4752/2016.

\section{References}

1. Towne, W.F., Kirchner, W.H.: Hearing in honey bees : detection of air-particle oscillations. Am. Assoc. Adv. Sci. 244(4905), 686-688 (1989)

2. Kirchner, W.H., Dreller, C., Towne, W.F.: Hearing in honeybees: operant conditioning and spontaneous reactions to airborne sound. J. Comp. Physiol. A 168(1), 85-89 (1991)

3. Collison, C.: A closer look: Sound generation and hearing. The Magazine of American Beekeeping (2016) 
4. Carreck, N.L., Andree, M., Brent, C.S., Cox-foster, D., Dade, H.A., Ellis, J.D., Hatjina, F.: Standard methods for Apis mellifera anatomy and dissection. J. Apic. Res. 52(4), 1-40 (2013)

5. Dreller, C., Kirchner, W.H.: Hearing in honeybees: localization of the auditory sense organ. J. Comp. Physiol. A 173(3), 275-279 (1993)

6. Service, H.B.: Bee anatomy (n.d.). https://sites.google.com/site/hutchingsbeeservice/home/ bee-anatomy. Accessed 15 Jan 2018

7. Hunt, J.H., Richard, F.-J.: Intracolony vibroacoustic communication in social insects. Insectes Soc. 60, 403-417 (2013)

8. Takeda, K.: Classical conditioned response in the honey bee. J. Insect Physiol. 6(3), 168179 (1961)

9. Menzel, R.: The honeybee as a model for understanding the basis of cognition. Nat. Rev. Neurosci. 13(11), 758-768 (2012)

10. Record, V., Devices, P.: ISD1700 Series. Pulse, pp. 1-24 (2006)

11. Webster, T.C.: Fumagillin affects Nosema apis and honey bees (Hymonopterai Apidae). J. Econ. Entomol. 87(3), 601-604 (1994)

12. Ellis, M.B., Nicolson, S.W., Crewe, R.M., Dietemann, V.: Hygropreference and brood care in the honeybee (Apis mellifera). J. Insect Physiol. 54(12), 1516-1521 (2008) 